\section{Tapering of Multitransmit Digital Beamforming Arrays}

Karin Schuler and Werner Wiesbeck

\begin{abstract}
Various taper functions are well known to suppress side lobes in antenna array factors. These tapers are limited to either transmit or receive arrays and are mostly restricted to arrays with a high number of elements. This paper presents an approach for applying taper functions also to digital beamforming (DBF) arrays with transmitter multiplexing. For such DBF configurations, taper functions have not been known yet. An example for a multiple transmit DBF taper will be given which is dedicated for low number of elements.
\end{abstract}

Index Terms-Digital beamforming (DBF), multitransmit digital beamforming, taper function.

\section{INTRODUCTION}

In contrast to phased arrays, digital beamforming (DBF) offers the possibility of focusing different angles simultaneously by exploiting multiple individually digitized channels. Since the main drawbacks of $\mathrm{DBF}$, the costs of multichannel AD-conversion and memory, are decreasing over time, and, on the other hand, the demand for imaging Radar also in consumer markets is grown, DBF becomes more and more important.

An extension to the common DBF on receive only is the use of multiple transmitters [1], [2]. With multiple transmit DBF, a better resolution can be achieved even when total number of elements in the transmit and receive array is the same as in the conventional case of DBF on receive-only. However, this leads to sparse transmit and sparse receive arrays. For them, the commonly used taper functions [3], [4] are not appropriate [5]. Also, a taper function applied to each array at once would not be sufficient since the linkage of transmitters and receivers during DBF processing is not taken into account.

In the following, DBF with multiple transmit antennas will be explained. Starting from this background, a derivation for an equivalent array will be presented. The equivalent array is a DBF system on receive-only. This allows the application of established taper functions. Finally, the conversion of the taper function backwards the multitransmit DBF will be illustrated by an example.

\section{Multiple Transmit Digital Beamforming}

In DBF-radar, the individual physical broad beams of all antennas overlap and cover a certain angular segment. The transmitter multiplexing can be achieved by sequentially switching distributed transmitters. On the receiving side, multiple coherent receivers collect the signals simultaneously. Each receiver input is digitized individually delivering the digital data for each transmit and receive antenna separately. This is an advantage of DBF and offers high flexibility for signal processing. The DBF signal processing generates from the digitized data a virtual narrow beam. This virtual beam can be moved in signal processing along the covered angular segment. Its beam therewith defines the angular resolution of the DBF-radar. For simplification, only

Manuscript received August 30, 2007; revised December 28, 2007. Published July 7, 2008 (projected).

The authors are with the Universität Karlsruhe (TH), Institut für Höchstfrequenztechnik und Elektronik (IHE), 76131 Karlsruhe, Germany (e-mail: karin. schuler@ihe.uka.de).

Color versions of one or more of the figures in this paper are available online at http://ieeexplore.ieee.org.

Digital Object Identifier 10.1109/TAP.2008.924774

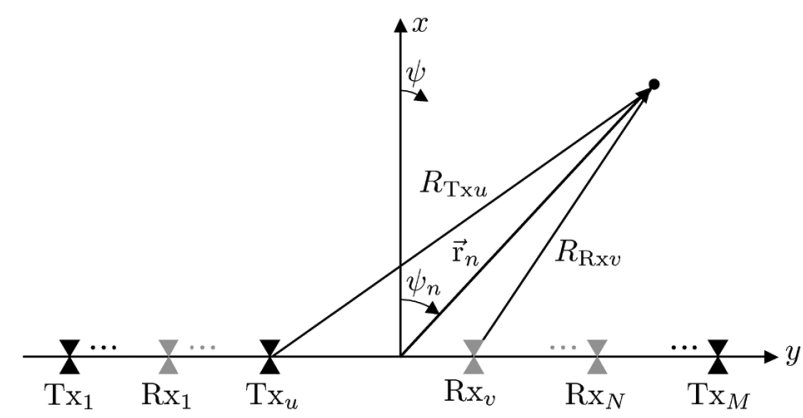

Fig. 1. Linear configuration of transmitters and receivers.

the phase centers of the individual antennas are considered in the following. $M$ transmit and $N$ receive antennas are placed along the $y$-axis. The position of a stationary reflecting object placed in the $x y$-plane is described by the range $\left|\overrightarrow{\mathbf{r}}_{n}\right|$ azimuth angle $\psi_{n}$ (see Fig. 1).

For azimuth compression, only the phase of the carrier signal $s_{\mathrm{R}}$ is important. The phase depends only on the propagation way and therewith the considered signal is

$$
s_{\mathrm{R}} \sim e^{j \frac{2 \pi}{\lambda}\left(R_{\mathrm{Tx} u}+R_{\mathrm{Rx} v}\right)} .
$$

Azimuth compression is realized as a multiplication of the received Radar signal with a test phase depending on the focussing angle $\psi_{0}$. The summation over all transmitter and receiver gives the azimuth compressed signal $f_{\mathrm{AC}}$

$$
f_{\mathrm{AC}}=\sum_{u=1}^{M} \sum_{v=1}^{N} k_{u, v} \cdot e^{-j \frac{2 \pi}{\lambda}\left(R_{\mathrm{Tx} u}+R_{\mathrm{Rx} v}\right)}\left[e^{-j \frac{2 \pi}{\lambda} K_{\mathrm{ac}}\left(\psi_{0}, u, v\right)}\right]^{*} .
$$

The important function is the kernel function $K_{\mathrm{AC}}$. It can be approximated by the phase that a target at a specific angle in the far field would evoke

$$
\begin{array}{r}
K_{\mathrm{AC}}\left(\psi_{0}, u, v\right)=-\left(x_{\mathrm{T} u}+x_{\mathrm{R} v}\right) \cos \left(\psi_{0}\right) \\
-\left(y_{\mathrm{T} u}+y_{\mathrm{R} v}\right) \sin \left(\psi_{0}\right) .
\end{array}
$$

In (2) $k_{u, v}$ is the taper function, which will be derived in Section III-B. Assuming a constant taper $\left(k_{u, v}=1\right)$, equidistantly spaced transmitters and receivers along the $y$-axes with the separation $\Delta y_{\mathrm{T}}$ and $\Delta y_{\mathrm{R}}$ and the object situated at the azimuth angle $\psi_{n}$, the azimuth compressed signal can be written as [2]

$$
\begin{aligned}
f_{\mathrm{AC}}=\frac{\sin \left(\frac{\pi M}{\lambda} \Delta y_{\mathrm{T}}\left(\sin \left(\psi_{n}\right)-\sin \left(\psi_{0}\right)\right)\right)}{\sin \left(\frac{\pi}{\lambda} \Delta y_{\mathrm{T}}\left(\sin \left(\psi_{n}\right)-\sin \left(\psi_{0}\right)\right)\right)} \\
\frac{\sin \left(\frac{\pi N}{\lambda} \Delta y_{\mathrm{R}}\left(\sin \left(\psi_{n}\right)-\sin \left(\psi_{0}\right)\right)\right)}{\sin \left(\frac{\pi}{\lambda} \Delta y_{\mathrm{R}}\left(\sin \left(\psi_{n}\right)-\sin \left(\psi_{0}\right)\right)\right) .}
\end{aligned}
$$

This is equivalent to the multiplication of two array factors [6]. This means that DBF with multiple transmitters delivers azimuth compression with the same performance as the multiplication of the array factors of the transmit and receive array. With that, also the side lobe level (SLL) is given by the multiplication of the two array factors. Usually, taper functions would be used to lower the SLL individually for the transmit and receive array. For multitransmit DBF this is not reasonable since a taper function would only affect the two arrays individually, but would neglect the combinations of transmit and receive antennas. 


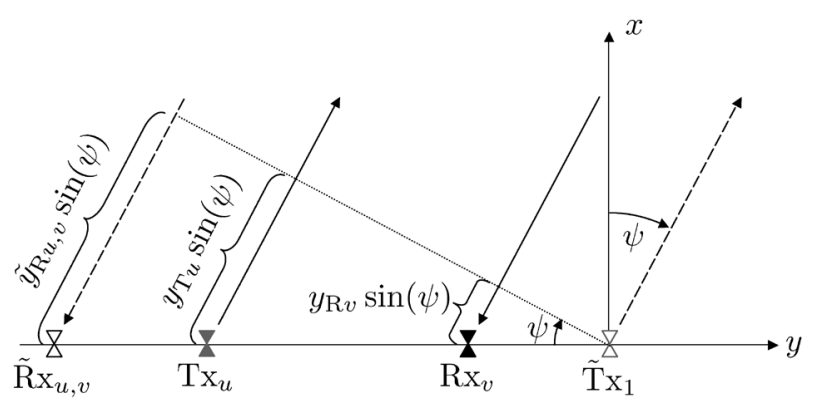

Fig. 2. Geometry for derivation of equivalent array.
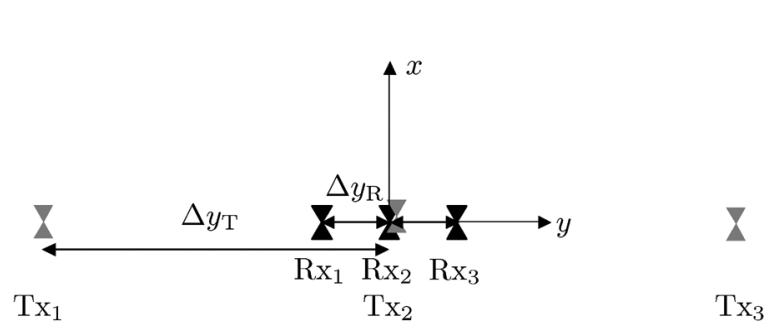

Fig. 3. Transmit and receive array.

\section{EQUIVALENT ARRAY}

To take into account the relationships of all transmit and receive antennas for a taper function, an equivalent array will be considered. This equivalent array consists of only one virtual transmitter $\tilde{\mathrm{T}} \mathrm{x}$ and $M N$ virtual receivers $\tilde{R} x$ placed along the $y$-axis [2]. To these virtual receivers a conventional taper can be applied.

\section{A. Determination of Equivalent Array}

For the determination of the equivalent array Fig. 2 illustrates the electric phase at the array.

The electric phase is referred to the origin of the coordinate system. The transmitter $\mathrm{Tx}_{u}$ introduces to its transmit signal the phase $\varphi_{\mathrm{Tx} u}$ relative to a transmitter which would be situated in the origin

$$
\varphi_{\mathrm{Tx} u}=y_{\mathrm{T} u} \sin (\psi) .
$$

For the receiver, the similar consideration is made. At the receiver $\mathrm{Rx}_{v}$ the phase $\varphi_{\mathrm{Rx} v}$ relative to a receiver at the origin is

$$
\varphi_{\mathrm{Rx} v}=y_{\mathrm{R} v} \sin (\psi) .
$$

To replace the physical transmit and receive antennas $\mathrm{Tx}_{u}$ and $\mathrm{Rx}_{v}$ by a virtual transmitter $\tilde{T} x$ situated in the origin and a virtual receiver $\tilde{\mathrm{Rx}} \mathrm{x}_{u, v}$, the total phase $\tilde{\varphi}_{\mathrm{Rx} u, v}$ relative to the origin must remain the same

$$
\begin{aligned}
\tilde{\varphi}_{\mathrm{Rx} u, v} & =\varphi_{\mathrm{Tx} u}+\varphi_{\mathrm{Rx} v} \\
\tilde{y}_{\mathrm{T} u, v} \sin (\psi) & =y_{\mathrm{T} u} \sin (\psi)+y_{\mathrm{R} v} \sin (\psi) \\
\tilde{y}_{\mathrm{R} u, v} & =y_{\mathrm{T} u}+y_{\mathrm{R} v} .
\end{aligned}
$$

The position of the virtual receiver $\tilde{\mathrm{R}} \mathrm{x}_{u, v}$ is therewith a function of the position of the associated transmitter $\mathrm{Tx}_{u}$ and receiver $\mathrm{Rx}_{v}$. The number of elements of the equivalent receive array is $M N$, the product of elements in the transmit and receive array.

For illustration, a multitransmit DBF double-array with $M=3$ transmitters and $N=3$ receivers is shown in Fig. 3. The equivalent array is shown in Fig. 4. In general, both the original arrays and the equivalent array are not equidistant.

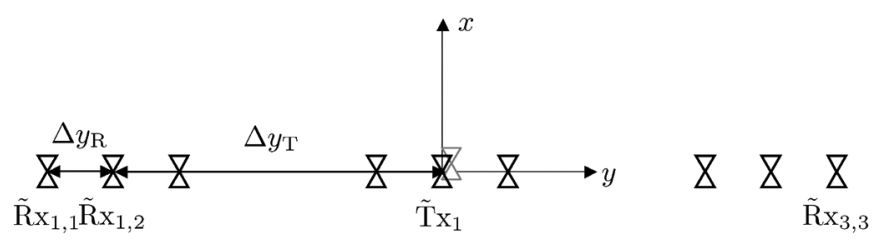

Fig. 4. Equivalent receive array.

TABLE I

TAPER COEFFICIENT VECTOR FOR EQUIVALENT ARRAY

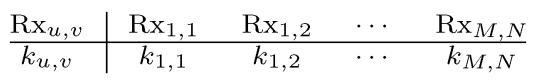

TABLE II

TAPER COEFFICIENT MATRIX FOR TRANSMIT AND ReCEIVE ARRAY

\begin{tabular}{c|cccc} 
& $\mathrm{Rx}_{1}$ & $\mathrm{Rx}_{2}$ & $\cdots$ & $\mathrm{Rx}_{N}$ \\
\hline $\mathrm{Tx}_{1}$ & $k_{1,1}$ & $k_{1,2}$ & $\cdots$ & $k_{1, N}$ \\
$\mathrm{Tx}_{2}$ & $k_{2,1}$ & $k_{2,2}$ & $\cdots$ & $k_{2, N}$ \\
$\vdots$ & $\vdots$ & $\vdots$ & $\ddots$ & $\vdots$ \\
$\mathrm{Tx}_{M}$ & $k_{M, 1}$ & $k_{M, 2}$ & $\cdots$ & $k_{M, N}$
\end{tabular}

TABLE III

VILLENEUVE-CoEfFicients $\left(N_{\text {equv }}=9, \mathrm{SLL}=40 \mathrm{~dB}, \tilde{n}=5\right)$

\begin{tabular}{c|ccccc}
$i$ & 1,9 & 2,8 & 3,7 & 4,6 & 5 \\
\hline$k_{i}$ & 0,1239 & 0,3451 & 0,6387 & 0,8981 & 1
\end{tabular}

\section{B. Application of Taper Coefficients to Multitransmit Array}

To the equivalent array, an arbitrary taper function can be applied. The taper coefficients $k_{u, v}$ are generally defined in Table I. When defining the taper coefficients $k_{u, v}$ for the equivalent receive antennas $\mathrm{Rx}_{u, v}$, their position $\tilde{y}_{\mathrm{R} u, v}$ must be considered.

To apply this taper to the multiple transmit DBF, it has to be transformed to the different combinations of transmitter and receiver. For this, the relation between virtual receiver and the corresponding transmitter and receiver according to (7) has to be used. This leads to a matrix of taper coefficients shown in Table II.

\section{EXAMPLE}

As an example, a multitransmit antenna configuration with both $M=N=3$ transmitters and receivers separated by $\Delta y_{\mathrm{R}}=0,6 \lambda$ and $\Delta y_{\mathrm{T}}=1,8 \lambda$ is considered. First, for the equivalent array different taper function are compared to illustrate the behavior of taper functions for sparse arrays. After that, one of these taper functions is applied in its matrix-form to a multitransmit DBF-Radar simulation. This approach points out the consistency of the array factor and the DBF azimuth compression, both weighted with the particular taper function in vector or matrix-form.

\section{A. Vector-Weighted Array Factor for Equivalent Array}

A Villeneuve taper function [7], [8] suitable for small arrays is applied to the equivalent array according to Fig. 4 consisting of $M N=9$ elements now equidistantly spaced with $\Delta y_{\mathrm{R} \text {,equv }}=0.6 \lambda$. The Villeneuve taper is the analogous of a Tayler taper for discrete arrays. The coefficients are given in Table III. Other taper functions are also possible, but since only a relatively small number of elements is available, the Villeneuve-taper gives better results.

In Fig. 5, the array factor is shown with an element spacing $\Delta y_{\mathrm{R}, \text { equv }}=0.6 \lambda$ and $N_{\text {equv }}=9$. With this single transmit antenna configuration, the array factor in Fig. 5 describes the theoretical azimuth compressed signal of the corresponding multitransmit DBF 


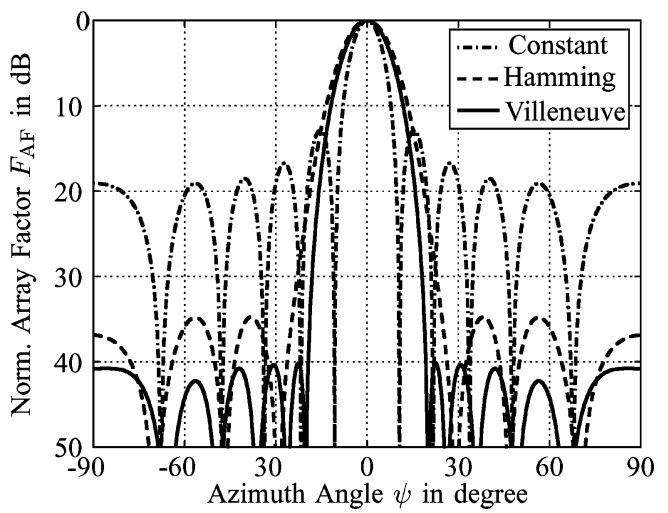

Fig. 5. Array factor of equivalent array. $\left(\Delta y_{\mathrm{R}}=0,6 \lambda, N_{\text {equv }}=9\right)$.

TABLE IV

TAPER COEFFICIENT MATRIX ACCORDING TO TABLE III

\begin{tabular}{c|ccc}
0 & $\mathrm{Rx}_{1}$ & $\mathrm{Rx}_{2}$ & $\mathrm{Rx}_{3}$ \\
\hline $\mathrm{Tx}_{1}$ & 0,1239 & 0,3451 & 0,6387 \\
$\mathrm{Tx}_{2}$ & 0,8981 & 1 & 0,8981 \\
$\mathrm{Tx}_{3}$ & 0,6387 & 0.3451 & 0.1239
\end{tabular}

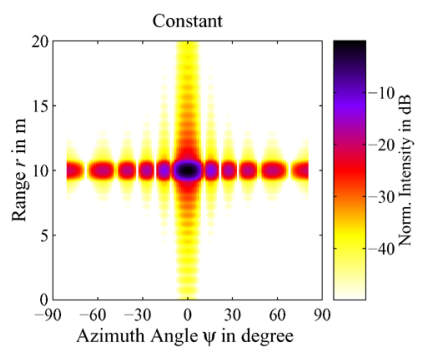

(a)

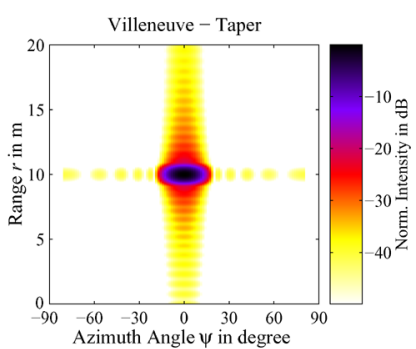

(b)
Fig. 6. Radar simulation of point target. ( $\Delta y_{\mathrm{R}}=0,6 \lambda, \Delta y_{\mathrm{T}}=1,8 \lambda, M=$ $3, N=3, B=250 \mathrm{MHz}$ ). (a) Constant taper. (b) Villeneuve taper.

array. The comparison shows that the chosen Villeneuve taper gives an even lower sidelobe level than the Hamming taper which does not reach the well-known sidelobe level of $-43 \mathrm{~dB}$ since the number of elements is to small.

\section{B. Matrix-Weighted Multitransmit DBF-Radar Simulation}

The taper coefficient matrix is given in Table IV. Only for the center transmitter, the receiver taper is symmetric. For the transmitter at the left, the taper coefficients for the receivers are increasing and for the transmitter at the right, the coefficients are decreasing.

A Radar simulation of a point target has been performed. A multi transmit DBF system has been assumed with transmitter and receiver distribution as described in Fig. 3. In Fig. 6(a) the simulated Radar image is given for constant amplitude distribution. In this case the sidelobe level is $-13 \mathrm{~dB}$. In Fig. 6(b) the same simulation has been performed with a Villeneuve distribution leading to a broader main lobe but a lower sidelobe level.

This simulation example confirms the applicability a taper matrix to multitransmit DBF systems.

\section{CONCLUSION}

In this paper, the application of linear taper functions to multitransmit DBF systems is derived. It shows that multitransmit DBF can be considered by an equivalent receive-only array. Arbitrary taper functions can now be applied to them and be converted to matrix form for multitransmit DBF systems. This offers the great advantage, that beneficial taper functions can be applied to these sparse double-arrays, where conventional taper functions would fail.

\section{REFERENCES}

[1] S. W. Smith, H. G. Pavy, and O. T. von Ramm, "High-speed ultrasound volumetric imaging system. I. Transducer design and beam steering," IEEE Trans. Ultrason., Ferroeletr., Freq. Control, vol. 38, no. 2, pp. 100-108, Mar. 1991.

[2] M. Younis, "Digital beam-forming for high resolution wide swath real and synthetic aperture radar," Ph.D. dissertation, Forschungsberichte aus dem Institut für Höchstfrequenztechnik und Elektronik der Universität Karlsruhe, Germany, 2004.

[3] F. J. Harris, "On the use of windows for harmonic analysis with the discrete Fourier transform," Proc. IEEE, vol. 66, no. 1, pp. 51-83, Jan. 1978.

[4] A. H. Nuttall, "Some windows with very good sidelobe behavior," IEEE Trans. Acoust., Speech Signal Process., vol. 29, no. 1, pp. 84-89, Feb. 1981.

[5] R. C. Hansen, Phased Array Antennas. New York: Wiley, 1997.

[6] C. Balanis, Antenna Theory: Analysis and Design. New York: Wiley, 1982.

[7] A. T. Villeneuve, "Taylor patterns for discrete arrays," IEEE Trans. Antennas Propag., vol. 32, no. 10, pp. 1089-1093, Oct. 1984.

[8] D. A. McNamara, "Generalised Villeneuve $\bar{n}$ distribution," Proc. Inst. Elect. Eng. Microwaves, Antennas and Propagation, vol. 136, no. 3, pp. 245-249, Jun. 1989.

\section{On a Class of Planar Absorbers With Periodic Square Resistive Patches}

\author{
Hosung Choo, Hao Ling, and Charles S. Liang
}

\begin{abstract}
A Pareto genetic algorithm is used to explore the performance of planar absorbers incorporating a sheet of periodic resistive patches embedded in a primary material substrate. The absorbing performance of a single-layer electric radar absorbing material (eRAM) with periodic resistive patches is compared to that of standard dual-layer absorbers made of wax laid on top of eRAM or magnetic radar absorbing material (magRAM).
\end{abstract}

Index Terms-Pareto genetic algorithm, periodic resistive patches, planar absorbers.

\section{INTRODUCTION}

The practical application of radar absorbers on aerospace vehicles can broadly be categorized into radar absorbing structures (RAS) [1], [2] and radar absorbing materials (RAM). RAS are primarily made of cellular core materials (loaded core or sandwiched loaded sheets) designed to handle partial structural load and are generally less than

Manuscript received July 10, 2006; revised January 9, 2008. Published July 7, 2008 (projected). This work was supported in part by the Office of Naval Research under Contract N00014-01-1-0224, in part by the Texas Higher Education Coordinating Board under the Texas Advanced Technology Program, and in part by the National Science Foundation Major Research Instrumentation Program.

H. Choo is with the School of Electronic and Electrical Engineering, Hongik University, Seoul, Korea (e-mail: hschoo@hiongik.ac.kr).

H. Ling is with the Department of Electrical Engineering, The University of Texas at Austin, Austin, TX 78712 USA.

C. S. Liang is with Lockheed Martin Aeronautics Company, Fort Worth, TX 76101 USA.

Color versions of one or more of the figures in this paper are available online at http://ieeexplore.ieee.org.

Digital Object Identifier 10.1109/TAP.2008.924766 